\title{
A few observations on Hermann Grid stimuli
}

\author{
Bernt Christian Skottun \\ Ullevaalsalleen $4 \mathrm{C}$ \\ 0852 Oslo \\ Norway \\ e-mail: berntchrskottun@gmail,com
}

Short title: Hermann Grid

\begin{abstract}
The Hermann Grid is made up of a series of vertical and horizontal bars. The Hermann Grid Illusion consists in the brightness of the intersections appearing different from that of the sections between intersections in spite of the luminance being the same. In the case of a light grid on a dark background the intersections tend to appear darker than the parts between intersections. It is here pointed out, in two different ways, that the stimulus power is less for the parts of the grid located at intersections than for parts of the grid between intersections. This is all in the stimuli and does not depend on vision or the visual system. Were we to assume that a stronger stimulus gives a brighter appearance this would make the parts between intersections appear brighter than the parts of the grid at intersections. This would be consistent with the Hermann Grid Illusion.
\end{abstract}

Key words: Illusion; Fourier Transform; amplitudes; stimulus power; interference. 


\section{INTRODUCTION}

The Hermann Grid Illusion (Hermann, 1870) can be obtained with a stimulus of the kind shown in Fig. 1A. The basic illusion consists in the brightness at the intersections appearing different from the brightness of the grid between the intersections in spite of the fact that the luminance of the grid is uniform throughout. In the case of a bright grid on a dark background (like the one shown in Fig. 1A) the brightness at the intersections tends to appear reduced relative to the brightness of the grid between the intersections.
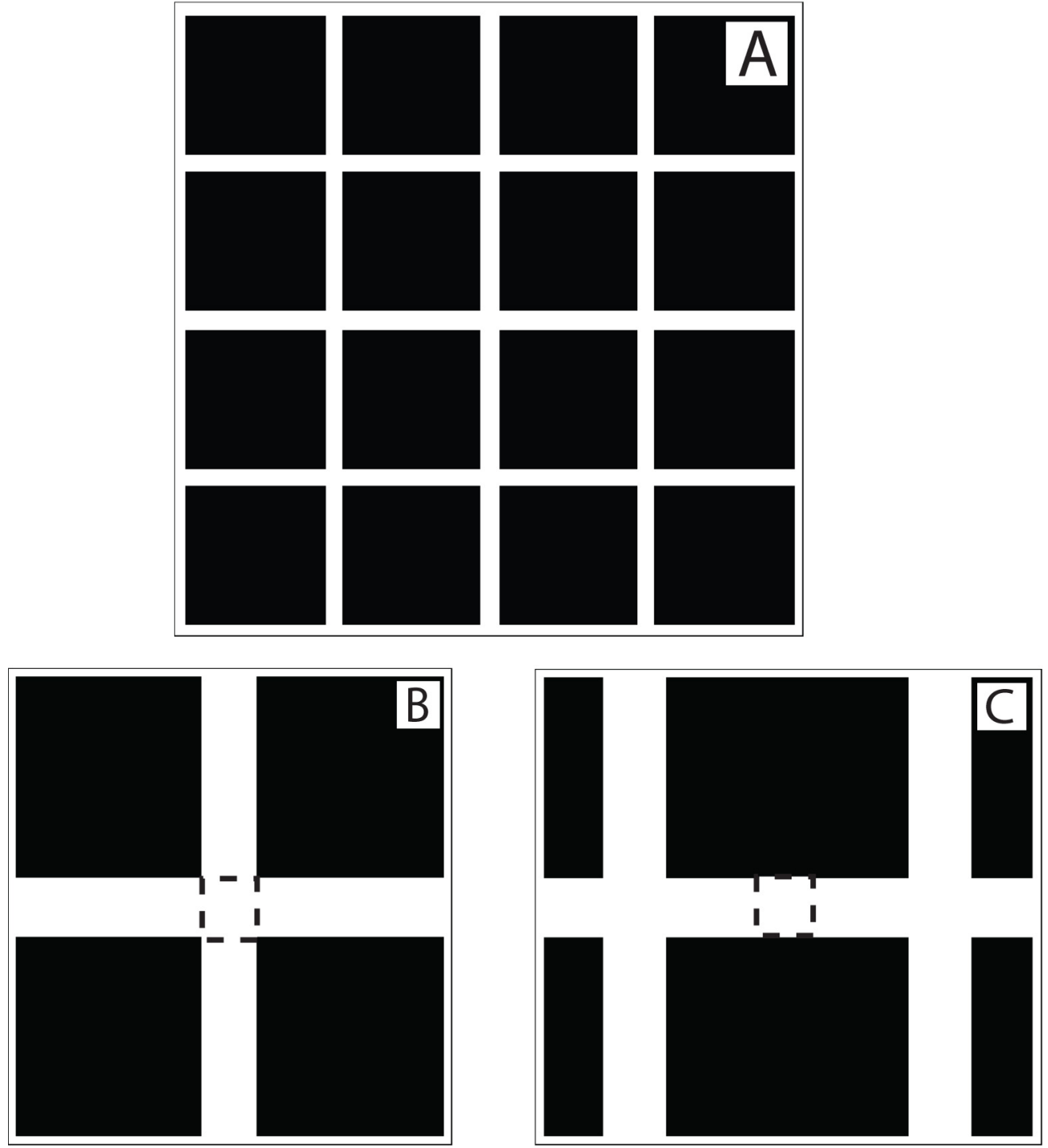

Fig. 1

Fig. 1. (A) An example of the Hermann Grid made up of a set of evenly spaced horizontal and vertical bright bars. The illusion consists in the part of the grid at the intersections appearing darker than the parts between intersections in spite of the fact that the grid has the same luminance throughout. (B) A square located at the intersection of two bright bars. (Outlined with a dashed line.) (C) A square located between the intersections of vertical and horizontal bars. (Outlined with a dashed line.) 
The Hermann Grid Illusion has been the topic of much interest (Hering, 1920; Baumgartner, 1960; Spillmann \& Levine, 1971; Lanvin \& Costall, 1978; Frisby, 1979; Levine et al., 1980; Troscianko, 1982; Lingelbach et al., 1985; Spillman, 1994; Palmer, 1999; Geier et al., 2004, 2008; De Lafuente \& Ruiz, 2004; Schiller \& Carvey, 2005; Hamburger et al., 2012; Johnston \& Timney, 2013; Roncato,et al., 2016; Bakshi \& Ghosh, 2020). Interpretations of this illusion have focused on the visual system. Specifically, they have tended to focus on center-surround organization of receptive fields such as found for retinal ganglion cells and LGN cells (Baumgartner, 1960; Schepelmann et al., 1967; Wolfe, 1984; Spillmann, 1994; Sekuler \& Blake, 1990; Goldstein, 2010; Kalat, 2016; Glover \& Lauzon, 2018). That is, they have largely ignored the stimuli. It seems reasonable to assume that in order to try to understand the responses to visual stimuli one ought to first understand the stimuli. The present analyses represent an attempt at that.

\section{ANALYSES}

For the present explorations the grid was light and the background dark as shown in Fig. 1A. Each vertical and horizontal bar, we may assume, is made up of squares (Fig. 1B and Fig. 1C; and Fig. 2 and Fig. 3). In which case we may take the illusion to consist in the squares at the intersections (Fig. 1B and Fig. 2) appearing darker than the ones located between intersections (Fig. 1C and Fig. 3). Thus considered the issue becomes one of determining the stimulus power of squares at these two locations. [FOOTNOTE 1]

The challenge then becomes to determine the stimulus power of identical squares in the two different contexts. That is, to obtain the stimulus power linked to one square in the grid without the inclusion of the stimulus power of other parts of the grid. This is a somewhat contradictory task in that we want to have the amplitudes linked to the square alone yet we want to have these as the square is in the context of the grid. I will here demonstrate two ways this may be approached.

\section{Analyses along the vertical and the horizontal dimensions.}

The first way is to restrict the analysis to the horizontal and vertical dimensions. If we accept that the grid is made up of a series of light squares on a dark background (see Figs. 1B and 1C) the question becomes one of determining the stimulus power (i.e., sum of amplitudes) associated with a square at an intersection (Fig. 1B) versus one located between intersections (Fig. 1C). Except for their locations in the grid these squares are identical. Their main characteristics and their main source of stimulus power is in their edges of which there are two vertical edges and two horizontal edges for each square. The task then becomes to 
determine the amounts of amplitudes associated with these edges. This is not completely straight forward since if one were to do this based on the Fourier Transform of the whole grid one would have included amplitudes associated with other vertical and horizontal contours in the grid.

To avoid this we compute the Fourier Transform separately for the horizontal and vertical dimensions. This can be done by computing the Fourier Transform by summing only the amplitudes linked to either the horizontal or vertical dimensions relative to the square. When determining the sum of amplitudes along the horizontal dimension we include only stimuli that are along the horizontal dimension of the square (Fig. 2B and 3B) and when calculating the sum of amplitudes for the vertical dimension we only include stimuli along the vertical dimension of the square (Fig. 2C and Fig. 3C). Were we to include elements along the vertical dimension when calculating the horizontal transform and elements along the horizontal dimension when computing the vertical transform these elements would have contributed to the transform and we would no longer have had only the amplitudes that can be linked to the square under study.

The analyses were carried out with a grid having $12 \times 12$ elements (Fig. 2A and Fig. 3A) and bars having a width of 1 making the squares have a side of 1 . The bright areas were set to a value of 1 and the dark background was set to 0 .

In the case of a square at an intersection we have that the sum of amplitudes along both the horizontal dimension (Fig. 2B) and the vertical dimension (Fig. 2C) are equal to zero when the d.c. values are not included. In the case of a square midways between intersections along a horizontal line (Fig. 3B) we also get zero for the horizontal dimension. But in the case of the vertical dimension (Fig. 3C) we need to compute the transform for a single square since this square does not have other bright squares along the vertical dimension either above or below it. When we do this we get the sum of amplitudes of 0.917. When adding all this we get zero amplitudes for a square at an intersection and a value of 0.917 for a square midways between intersections. That is, a square midway between intersections has more stimulus power than one located at an intersection. 

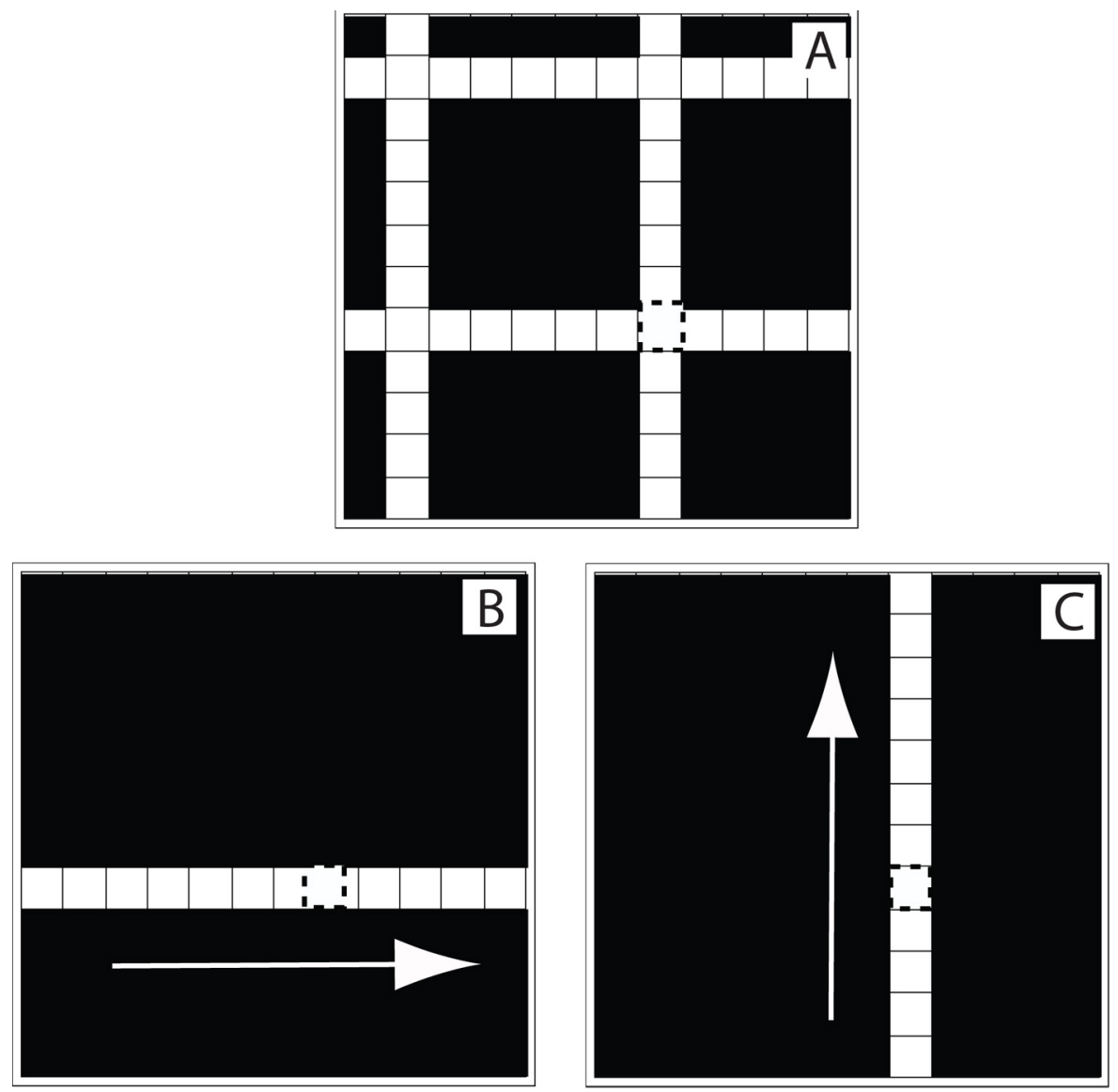

Fig. 2

Fig. 2. Analysis of a square located at the intersection of a vertical and a horizontal bar. (A) The analyzed square is indicated with a dashed line. (B) Analysis along the horizontal dimension. (C) Analysis along the vertical dimension. The size of each square was $1 \times 1$. In both cases the sum of the amplitudes (after the d.c. values have been set to zero) is zero. The white arrows indicate the directions for which the analyses were carried out.

Were we to include the d.c. values we would be adding 2 times the d.c. value for a single square (i.e., $2 \times 0.0833=0.1667)[$ FOOTNOTE 2] to the two values above giving us a total on 0.1667 for squares at intersections and 1.0833 for squares midways between intersections. [FOOTNOTE 3] Thus, irrespective of whether or not we include the d.c. values we find that a square midways between intersections has more stimulus power than one located at an intersection. If we make the assumption that a larger stimulus power makes the stimulus appear brighter this would make the areas between intersections appear brighter than areas at intersections. This would be consistent with the Herman Grid Illusion for a bright grid on a dark background. 

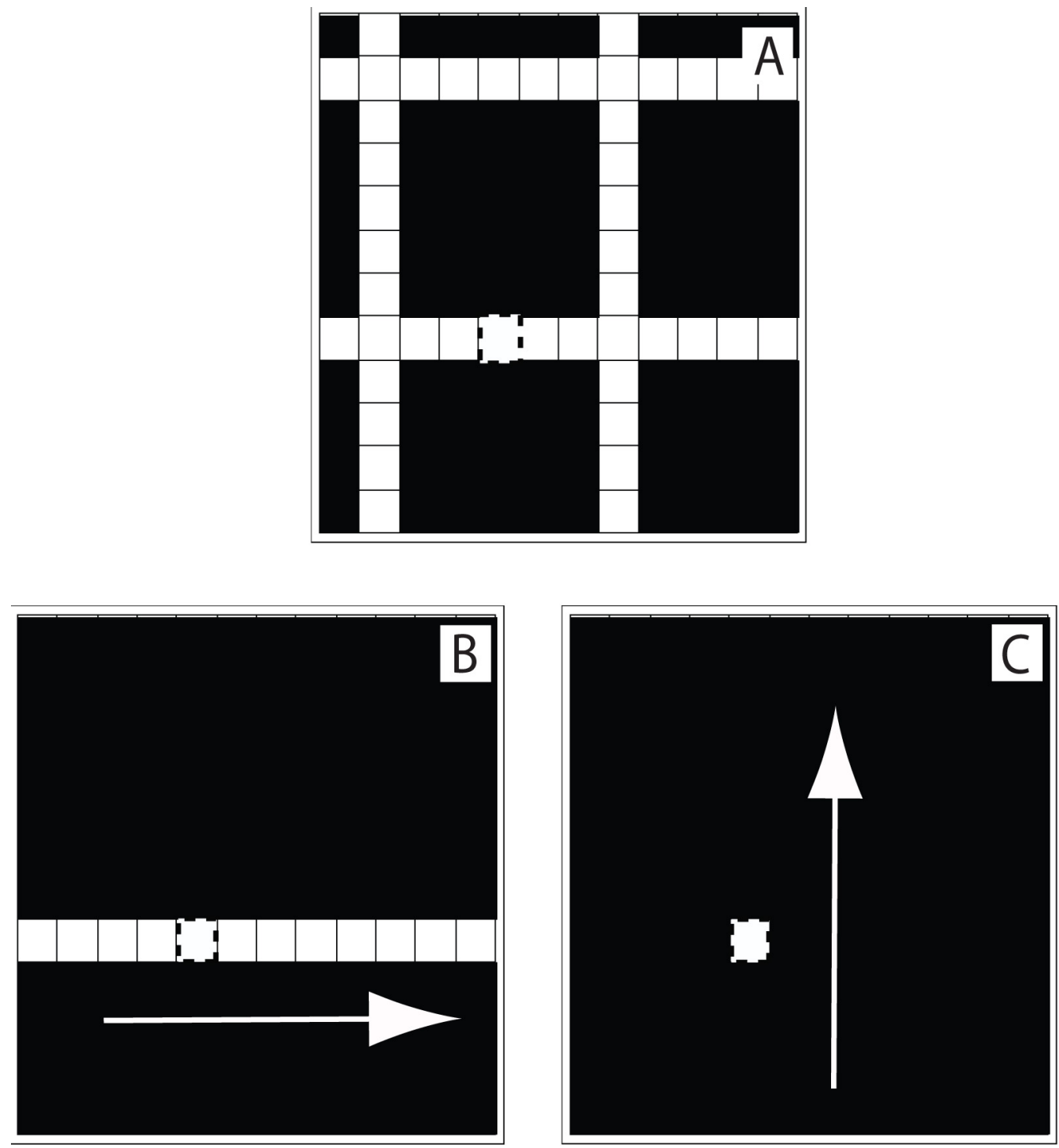

\section{Fig. 3}

Fig. 3. Analysis of a square located between the intersection of a vertical and a horizontal bar. (A) The analyzed square is indicated with a dashed line. (B) Analysis along the horizontal dimension. (C) Analysis along the vertical dimension. In the case of analysis along the horizontal dimension (Panel B) the sum of the amplitudes (after the d.c. value has been set to zero) is zero. In the case of analysis along the vertical dimension (Panel C) the sum of the amplitudes (after the d.c. value has been set to zero) is 0.917 . The white arrows indicate the directions for which the analyses were carried out.

The Hermann grid Illusion can also be obtained with a dark grid on a bright background. In which case we simply need to flip the polarity and we get the same result. The only difference is that instead of brightness we now have darkness and detectors of increments of light will now have to be replaced by detectors of decrements. That there exist detectors that respond to either increments or decrements (ONor OFF center cells) and even cells that respond to both (cortical complex cells) is well established. 


\section{Multiplication with a Gaussian.}

Another way to approach the issue is to confine the analysis to only the region immediately around the square in question. By doing so one may minimize the inclusion of the amplitudes linked to contours outside of the square while at the same time taking account of the context in which the square is located. This can be done by multiplying the stimulus with 2-D Gaussian centered upon the square to be analyzed.

The stimuli used with this procedure are illustrated in Fig 4. Each image is made up of $256 \times 256$ image elements with bright bars having a width of 33 elements. Consequently a square is made up of $33 \mathrm{x}$ 33 elements. The stimuli have all been multiplied with a 2-D Gaussian. In the present analyses a square (Fig. 4A) was analyzed with (1) a bar on either side (Fig. 4B). This would correspond to a square located between intersections (Fig. 4D). Or alternatively, (2) with four bars abutting bars (Fig. 4C). This would correspond to a square at an intersection (Fig. 4E). The question then becomes is the sum of amplitudes for a square between intersections (i.e., as shown in Fig. 4D) larger than for one at intersections (i.e., as in Fig. 4E)? (The analysis was carried out with a Gaussian having a $\sigma=8$ elements. For the sake of illustration the stimuli in Fig. 4 have been multiplied with a Gaussian having a $\sigma=20$. This is because the stimuli multiplied with a Gaussian having $\sigma=8$ elements made the pictures of the surrounding stimuli, i.e. Fig. 4B and Fig. 4C, very faint and made it difficult to tell Fig. 4D and Fig. 4E apart.)

The sum of amplitudes for a square between intersections (i.e., as shown in Fig. 4D) is 282 and for a square at intersections (i.e., as shown in Fig. 4E) it is 262. Thus, we have that the sum of amplitudes for a square at an intersection is smaller than for one between intersections. For comparison, the sum of amplitudes for the square alone is 311 . Thus, for both a square between intersections and one at intersections the sum of amplitudes is less than for the square in isolation. However the reduction is larger for the square at an intersection than for one between intersections.

Some may perhaps ask how adding something to a stimulus element may cause the sum of its amplitudes

to be reduced. The answer is with the concept of interference. "Interference" refers to the fact that the amplitude of the sum of two Fourier components is smaller than the sum of the amplitudes of the two components determined separately when these differ in phase angle (Skottun, 2018a, b, c). Interference takes place in the stimuli and is independent of them being seen or them activating any visual system. 

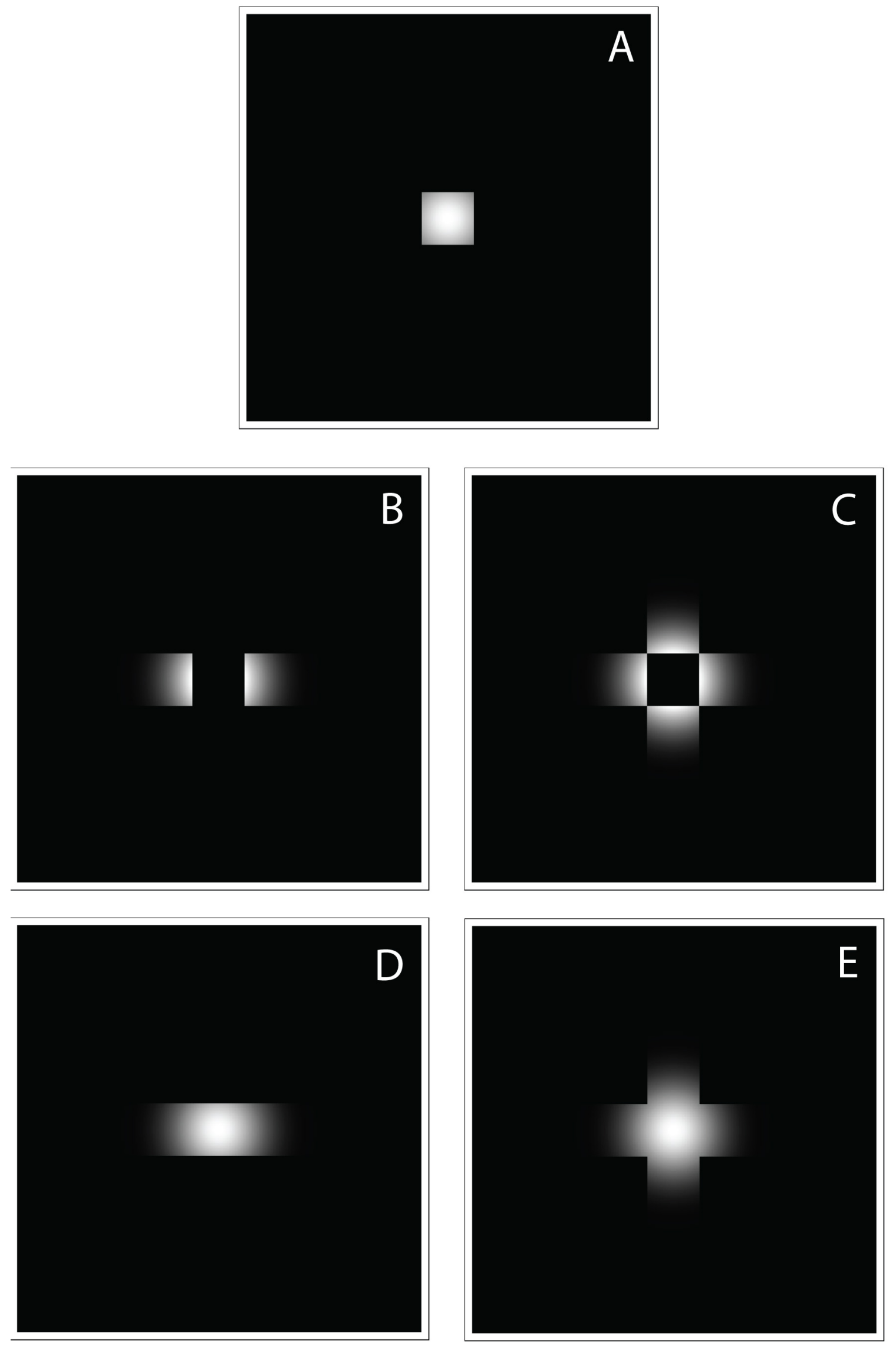

Fig. 4 
Fig. 4. The stimuli multiplied with a Gaussian. (A) A single square multiplied with a Gaussian. (B) The two adjacent light bars for a square located between intersections. (C) The four light bars adjoining a square at an intersection. (D) A square between intersections along with its adjoining two bars (i.e., Fig. 4A + Fig. 4B). (E) A square at an intersection along with its four adjoining bars (i.e., Fig. 4A + Fig. 4C). The bar had a width of 33 elements giving squares of $33 \times 33$ elements. Each of the images spanned $256 \times 256$ elements. Although the analyses were carried out with a Gaussian having a $\sigma=8$ picture elements it is here, for the sake of clarity, shown with a Gaussian having a $\sigma=20$ elements.

Interference may be quantified by the Relative Amplitude Sum (Skottun, 2018a, b, c). For two 2-D Fourier spectra, A and B, we may define the Relative Amplitude Sum as

$\sum_{x} \sum_{y}|(a+b)| /\left(\sum_{x} \sum_{y}|a|+\sum_{x} \sum_{y}|b|\right)$ where $a$ and $b$ are the components in the Fourier spectra $A$ and $B$, respectively, with |.| denoting the amplitude operator. The Relative Amplitude Sum is the sum of amplitudes in the combined stimulus, $(\mathrm{A}+\mathrm{B})$, relative to the sum of amplitudes in A plus the sum of amplitudes in B. If there is no interference the Relative Amplitude Sum will be 1.0. Any reduction below 1.0 indicates interference and the amount of reduction below 1.0 can be taken as a measure of the amount of interference. It was found that the Relative Amplitude Sum were 0.66 and 0.79 for squares at intersections and squares between intersections, respectively, indicating more interference at intersections than between them.

Since the Relative Amplitude Sum gives the amplitudes in two (or more) stimuli determined together relative to them being determined separately one cannot attribute the reductions caused by interference to one of the two (or more) interfering stimuli. However, in the present case since the sum of amplitudes for the square along with the surround is smaller than that of the square alone (i.e., were reduced from 311 to 282 and from 311 to 262 for squares between the intersections and at intersections, respectively) it is apparent that the amplitudes of the squares were reduced and that the reduction was larger for a square located at an intersection than for one located between intersections.

If we assume that out of two identical stimuli the one that is more reduced by interference (i.e., has the lower Relative Amplitude Sum) will appear less bright we have that in the case of a bright Hermann Grid on a dark background the square at an intersection will appear less bright than one located between intersection, which is what is experienced with a bright Herman Grid on a dark background.

\section{CONCLUSIONS}

The present analyses have documented quantitatively that a square at the intersection of two lines in the Hermann Grid has less stimulus power than one located between intersections. That this is the case ought 
to have been obvious from just looking at the stimuli since a square with two edges of high contrast has more stimulus power than one that has none.

If we assume (in the case of a bright stimulus on a dark background) that a stimulus with higher stimulus power will appear brighter than one with less power, in other words if we assume that a stronger stimulus appears brighter, it would follow immediately that the parts of the grid between intersections of the vertical and horizontal bars will appear brighter than the ones located at intersections. That a stronger stimulus should give rise to a brighter appearance seems a reasonable assumption. Based on the present observations and this assumption it is puzzling how the Hermann Grid Illusion could have become the topic of much interest among psychologists.

The present analyses rest fundamentally upon the stimuli. The only assumption made about the visual system is that a stimulus with more stimulus power may result in a brighter appearance. It is usually held that the most parsimonious explanation is to be preferred. It seems that an explanation that does not require the involvement of the visual system, or does so only minimally, is more parsimonious than one that depends on both the stimuli and the visual system.

Given that it may be possible to understand the basic Hermann Grid Illusion on the basis of the stimuli (other than the trivial assumption that a stronger stimulus results in a brighter appearance) it seems this illusion can tell us little about vision and the visual system. Although it is difficult to see that the basic Hermann Grid Illusion requires explanations in terms of the visual system some aspects of this illusion may do so. It may be that what requires explantions based on the visual system may not be why we experience the Herman Grid Illusion but rather why we do not see it under some special conditions, e.g. when tilted (Wolfe, 1984 ) or when the lines making up the grid are bent (Geier et al., 2008). In this connection it should be pointed out that Bakshi and Gosh (2020) found that small Squares at the corners in the Hermann Grid can remove the illusion. This may be consistent with the present interpretation since the small squares would increase the stimulus power at the intersections by increasing the amount of edges at these locations. This could cause the apparent brightness at the corners of the grid to be increased.

It seems therefore that the basic Hermann Grid Illusion can be understood based on the stimuli. The Hermann Grid Illusion may not be alone in this regard since there is evidence to indicate that the Tilt Illusion may be understood in terms of interference between two elongated stimulus elements (Skottun, 2018d; 2020). There are also indications that the Ponzo illusion may be understood mainly based on interference in the stimuli (Skottun, 2019). However, in this case one needs to make the assumption that the visual system 
integrates stimulation within only limited areas. To the extent these illusions may be understood based on the stimuli it would seem they will tell us little about vision or the visual system.

The Introduction pointed out the need to understand visual stimuli. Such understanding may require the stimuli be re-represented with different basis functions (e.g., be re-represented by the Fourier Transform or a wavelet transform). For instance, Skottun et al. (1994) showed that the direction selectivity of cortical cells to rapidly drifting dots, which had been considered to represent a problem, became obvious once the stimuli were re-represented in a 3-D spectral space (i.e., a 3-D Fourier space). Thus, we may say that what appeared as a "problem" was the result of describing the stimuli in an inadequate fashion. In the case of the Herman Grid Illusion the notion of a problem, or of something which was judged to require an explanation, rested on the fact that in the space domain the grid physically has uniform luminance whereas the luminance appears unequal in different parts of the grid. However as shown here, this is not the only possible way of describing these stimuli. When recast in terms of the amplitudes in the Fourier Transform linked to different parts of the grid the "problem" disappears. We may be inclined to say that what was taken to be an illusion, or a "problem", rested on describing the stimuli in a particular way.

\section{FOOTNOTES.}

1. For a discussion of "stimulus power" see Skottun, (2018c).

2. The d.c. value of a single square with an intensity value of 1 in a $12 \times 12$ elements grid becomes $0.0833=1 / 12$ since the grid in this analysis has 144 elements The scaling factor for the Fourier Transform is the inverse of the square root of the number of elements. In the present case this gives us $1 / \sqrt{144}=1 / 12$.

3. Were we to carry out the analyses for a larger grid with larger bars and squares the specific number would be different but the overall result would be the same. For instance, with a $256 \times 256$ elements image with a bar width of 16 we get amplitudes sums without the d.c. values of 35.2 and 0 for a (16 x 16 elements) square between intersections and at the intersections, respectively. Were we to add the d.c. value for the square we would get 37.5 and 2.3 for squares at the sides and at the intersections, respectively. 


\section{REFERENCES}

Bakshi, A. \& Ghosh, K. (2020) Tiny squares at the Hermann grid corners can completely remove the illusion. Perception, 49, 232-239.

Baumgartner, G. (1960) Indirekte Grossenbestimmung der rezeptiven Felder der Retina beim Menschen mittels der Hermannschen Gittertauschung. Pflugers Archiv fur die gesamte Physiologie, 272, 21-22.

De Lafuente, V. \& Ruiz, O. (2004) The orientation dependence of the Hermann grid illusion. Experimental Brain Research, 154, 255-260.

Frisby, J.P. (1979) Seeing-Illusion, Brain and Mind. Oxford University Press. Oxford, UK.

Geier. J., Sera, L. \& Bernath L, (2004) Stopping the Hermann grid illusion by simple sine distortion. Perception, 33, Supplement, 53.

Geier, J., Bernath, L., Hudak, M. \& Sera, L. (2008) Straightness as the main factor of the Hermann grid illusion. Perception, 37, 651-665.

Glover, E.M. \& Lauzon, O. (2018) Using a contrast illusion to teach principles of neural processing. The Journal of Undergraduate Neuroscience Education, 17, A81-A88.

Goldstein, E.B. (2010) Sensation and perception. Wadsworth, Belmont, CA, USA.

Hamburger, K., Baier, F., \& Spillmann, L. (2012) The tilted Hermann grid illusion: 'Illusory spots' versus 'phantom bands'. Perception, 41, 239-242.

Hering, E., (1920) Vom simultanen Grenzkontrast. In Grundzuge der Lehre vom Lichtsinne. Chapter 32 (Berlin: Springer) [Outlines of the Theory of the Light Sense; translation by L M Hurvich, D. Jamieson (1964, Cambridge, MA: Harvard University Press)].

Hermann, L. (1870) Eine Ersheinung des simultanen Contrastes. Pflugers Archiv Fur Die Gesamte Physiologie, $3,13-15$.

Johnston, K. \& Timney, B. (2013) Alcohol and lateral inhibitory interactions in human vision. Perception, $42,1301-1310$.

Lanvin, E. \& Costall, A. (1978) Detection thresholds of the Hermann Grid Illusion. Vision Research, 18, 1061-1062.

Levine, J., Spillmann, L. \& Wolf, E. (1980) Saturation enhancement in colored Hermann Grid varying only in chroma. Vision Research, 20, 307-313.

Lingelbach, B., Block, B., \& Hatzky B, \& Reisinger, E. (1985) The Hermann grid illusion retinal or cortical?' Perception, 14(1), A7 (abstract).

Kalat, J.W. (2016) Biological Psychology. Cengage Learning: Boston, MA. USA.

Palmer, S.E. (1999) Vision Science-Photons to Phenomenology. The MIT Press, Cambridge, MA, USA.

Roncato, S., Guidi, S., Parlangeli, O. \& Battaglini, L. (2016) Illusory streaks from corners and their perceptual integration. Frontiers in Psychology, 7, 959.

Schepelmann, F., Aschayeri, H. \& Baumgartner, G. (1967) Die Reaktionen der 'simple field'-Neurone in Area 17 der Katze beim Hermann-Gitter-Kontrast. Pflugers Archiv fur die gesamte Physiologie, 294 R57 (Abstract).

Schiller, P.H. \& Carvey, C.E. (2005) The Hermann grid illusion revisited. Perception, 34, 1375-1397. 
Sekuler, R. \& Blake, R. (1990) Perception. 2nd Edition. McGraw-Hill Publishing Company: New York:, NY, USA.

Skottun, B.C. (2018a) Interference in lateral masking stimuli-The effects of relative phase, position, orientation, and spatial frequency. Behavioral Brain Research, 349, 137-144.

Skottun, B.C. (2018b) A few remarks on spatial interference in visual stimuli. Behavior Research Methods, $50,1716-1722$.

Skottun, B.C. (2018c) A few remarks on stimulus power and interference in visual stimuli. PsyArXiv, doi: 10.31234/OSF.IO/3NT84.

Skottun, B.C. (2018d) Is the Tilt-Illusion in the stimuli? PsyArXiv. DOI: 10.31234/osf.io/mwxvd

Skottun, B.C. (2019) A few observations on the amplitude spectra of Ponzo Illusion stimuli. PsyArXiv. DOI: $10.31234 /$ osf.io/96nxt

Skottun, B.C. (2020) Interference in Tilt-Illusion stimuli: A simple illustration. PsyArXiv. DOI: 10.31234/osf.io/qw3dt

Skottun, B.C., Zhang, J. \& Grosof, D.H. (1994) On the directional selectivity of cells in the visual cortex to drifting dot patterns. Visual Neuroscience, 11, 885-897.

Spillmann, L. (1994) The Hermann grid illusion: a tool for studying human perspective field organization. Perception, 23, 691-708.

Spillmann, L. \& Levine J. (1971) Contrast enhancement in a Hermann grid with variable figure-ground ratio. Experimental Brain Research, 13, 547-559.

Troscianko, T. (1982) A stereoscopic presentation of the Hermann Grid. Vision Research, 22, 485-489.

Wolfe, J.M. (1984) Global factors in the Hermann grid illusion. Perception, 13, 33-40. 